\title{
Simuladores para el mejoramiento de las competencias de pensamiento estratégico en la Administración
}

\section{Simulators for Improving Strategic Thinking Competencies in Management}

\author{
Frederick Mendoza-Lozano \\ Ronald Martínez-Contreras \\ Luis Trujillo-Flórez \\ Nicolás Arias-Velandia \\ Ángela Ríos-Gallardo \\ Institución Universitaria Politécnico Grancolombiano, Bogotá, Colombia
}

\section{Resumen}

Objetivo: Fortalecer las competencias de análisis prospectivo y diagnóstico empresarial a través de herramientas de aprendizaje inmersivo desarrolladas por la Institución Universitaria Politécnico Grancolombiano, en los estudiantes de la modalidad virtual. Método: El laboratorio de experiencias de aprendizaje inmersivo desarrolló una metodología fundamentada en el referente ADDIER (Analysis, Desing, Development, Implementation, Evaluation, Research) con la que se hizo el diseño e implementación de dos ambientes inmersivos. A través de una encuesta, se presenta la percepción de los estudiantes sobre el impacto de estas herramientas en su proceso formativo. Resultados: Los estudiantes expresaron un alto nivel de satisfacción asociado a la eficiencia y eficacia de su proceso formativo, derivado del uso de los ambientes inmersivos. Específicamente, se reportaron valoraciones positivas en ahorro de tiempo, minimización de errores, mejora en la calidad de los análisis empresariales y retroalimentación del tutor. Discusión y Conclusiones: Un rasgo innovador de esta propuesta es que permite que se trabaje con base en experiencias reales y no en datos simulados. También se concluye que el éxito de esta experiencia se debe al trabajo de los tutores, en el sentido de su apertura y dedicación para la introducción de herramientas innovadoras en los cursos de educación virtual.

Palabras clave: Ambientes inmersivos de aprendizaje, Diseño curricular, Pensamiento estratégico, Simuladores.

\begin{abstract}
Purpose: To reinforce business prospective and diagnostic analysis competencies in online students using immersive learning tools developed by the Politécnico GranColombiano University Institution. Method: The Immersive Learning Experience Lab developed a methodology based on the ADDIER (Analysis, Design, Development, Implementation, Evaluation and Research) model, which was used to design and implement two immersive environments. Student perception of the impact of these tools on their educational process is presented through a survey. Findings: Students state that they are highly satisfied with the efficiency and effectiveness of their educational process after using immersive environments. Specifically, positive assessments are reported in terms of time savings, error minimization, quality improvement in business analysis, and feedback from the instructor. Discussion y Conclusion: The innovation of this proposal lies in the fact that it supports working from real experiences instead of from simulated data. The study also concluded that this experience was successful owing to the dedication of instructors in terms of their openness and willingness to introduce innovative tools in online education courses.
\end{abstract}

Keywords: Immersive learning environments, degree program design, strategic thinking, simulators.
Open Access:

ISSN: $0124-2121$

E-ISSN: $2665-2420$

ARTÍCULO RESULTADO DE INVESTIGACIÓN Copyright ( 2020 By Educación y Humanismo

Editor:

Patricia Martínez Barrios Universidad Simón Bolívar

Recibido: 03-10-2019 Aceptado: 13-01-2020 En línea desde: 08-04-2020 


\section{Introducción}

El uso de estrategias de simulación en ambientes educativos es antiguo, tanto como la intención de acercar el trabajo del aula con la realidad que le espera a los futuros graduados (Stapleton, 1990); y se ha vuelto relevante en el aprendizaje de las ciencias administrativas, en tanto ellas concentran en Colombia, la mayor parte de los estudiantes que aspiran a desempeñarse eficientemente en ambientes laborales específicos. El programa académico de Administración de Empresas de la Institución Universitaria Politécnico Grancolombiano (IUPG) cuenta con el mayor número de estudiantes en la institución: para el segundo semestre de 2018 registró un total de 10.120 matriculados, de los cuales el $90 \%$ son de la modalidad virtual. Entre tanto, el Sistema Nacional de Información de la Educación Superior (SNIES), en los programas nacionales de Administración de Empresas, registró un total de 463.878 matriculados en nivel de pregrado, de los cuales, el $31.43 \%$ está en modalidad virtual o a distancia.

Un volumen tan grande de estudiantes en el área de ciencias administrativas evidencia la necesidad de establecer diferenciales entre la oferta. En ese sentido, un camino recomendable es innovar en la didáctica, con orientación al "aprender haciendo", para que los egresados de los programas sean capaces de realizar consultorías empresariales, lo que implica un cambio en la planeación curricular y la adquisición de competencias de pensamiento estratégico. Con esa motivación, el propósito de este trabajo es aportar en el ámbito de las herramientas innovadoras para la didáctica de la administración, haciendo uso de simuladores virtuales.

Uno de los problemas que presenta en la educación actual se fundamenta en cómo adquirir y evaluar competencias en contextos situados. Algunos estudios resaltan la importancia de vincular el currículo con los problemas propios del mundo real (Klink \& Boon, 2002; Smyth \& Dow, 1998; Tchibozo, 2008). Para la IUPG, las prácticas aplicadas permiten combinar una experiencia educativa con el contexto real; además, constituye una alianza virtuosa entre la empresa y las Instituciones de Educación Superior (Franco, Silva, \& Rodrigues, 2019), donde el estudiante pone en práctica los conocimientos adquiridos y evidencia la apropiación de las competencias en sus tres dimensiones: saber, hacer y ser.

Sin embargo, conseguir espacios ideales para que los estudiantes realicen sus prácticas no es fácil; por eso, en esta investigación se planteó una opción diferente, donde se emplean Ambientes Inmersivos (AI) en los que el aprendiz tiene una alta implicación no sólo cognitiva sino sensorial y motivacional (Dede, 2009). El propósito es que el estudiante tenga mayor implicación y un aprendizaje más profundo, por lo tanto, trabaja en una empresa real de donde extrae información primaria; el AI le ayuda en sus análisis y a partir de eso, realiza la planeación estratégica de una empresa. 
Motivado en lo anterior, en la IUPG se implementó dos AI realizados por el Laboratorio de Experiencias de Aprendizaje Inmersivo (LEAI) con una estrategia educativa que tiene dos componentes: uno pedagógico y otro tecnológico. En el primero, se trabajó desde el aprendizaje situado y con la metodología activa basadas en problemas. En el segundo, se diseñaron y desarrollaron dos softwares para aplicarse en ambientes empresariales: uno para el análisis prospectivo y otro para el diagnóstico estratégico.

Los avances tecnológicos permiten crear ambientes inmersivos fácilmente y a bajo costo (Neri, Noguez, Pérez, \& Aguilar, 2011). De igual manera, la estrategia de inmersión abarca las siguientes dimensiones: 1) Lo didáctico-curricular con una ruta de aprendizaje estructurada, alternativas y caminos diseñados para que el estudiante ponga en evidencia sus competencias. 2) Aumento de la motivación y el interés del estudiante. 3) Estrategia de evaluación que involucra procesos como la toma de decisiones, la argumentación y la comprensión de contextos complejos. Lo anterior implica que el docente asuma una forma alternativa de enseñanza, acompañamiento y evaluación, coherente con los componentes pedagógicos de las herramientas inmersivas (Báez, Manzouli, Cifuentes, Duarte, Otero y Rees, 2012).

EI LEAI construye diversos ambientes inmersivos de acuerdo con las necesidades de los programas según la matriz de competencias que expone los cursos en los que se involucra cada una; y los perfiles profesionales. Este proyecto se construye mediante una alianza entre el LEAI y la Escuela de Administración y pretende que los estudiantes sean capaces de pensar de manera estratégica en un proceso de consultoría como lo propone Drucker, 1974 citado en Téllez, 2005): "visualizar el negocio por completo, tomar decisiones que lo afecten, evaluar objetivos y necesidades actuales y futuros y que pueda distribuir los recursos humanos y monetarios para obtener resultados claves" (p.116).

\section{Diseño de la experiencia de aprendizaje}

Como se había mencionado, el propósito es fortalecer el pensamiento estratégico en los estudiantes cuando realizan consultorías empresariales. Por tal motivo, se implementó: 1. Un proceso de fortalecimiento curricular que compila estrategias inmersivas en diversos cursos, de modo que cuando el estudiante llegue a los semestres avanzados, sus habilidades y aptitudes en pensamiento estratégico estén listas para afrontar una consultoría real. 2. Se plantea un trabajo de acompañamiento a empresarios por parte de los estudiantes quienes a su vez son asesorados por los profesores. La IUPG hace un convenio de consultoría con las empresas donde se comprometen a entregar la información a los estudiantes; estos analizan la información y realizan un plan estratégico con recomendaciones y orientaciones con el fin de que la empresa lo lleve a cabo. 3. Se culmina con el diseño, desarrollo e implementación de AI que permitan fortalecer las competencias de análisis estratégico. En dichos ambientes los estudiantes ingresan la información y realizan un análisis prospectivo y un diagnóstico, que a su vez les sirve de insumo para elaborar un plan estratégico robusto. 
El proceso inicia con el diseño pedagógico de la experiencia de aprendizaje, la cual propone que, de manera colaborativa, los estudiantes analicen empresas y generen estrategias de planeación. Para ello, deben identificar los factores críticos de éxito; con el diagnóstico visualizan el presente y con el análisis prospectivo prevén el futuro de la empresa; de esa manera, obtienen los insumos para elaborar el plan estratégico. Tal diseño parte de la primicia que propone Tobón (2006): el estudiante debe ser capaz de resolver problemas reales y elaborar productos o servicios que son importantes para la comunidad. Y se complementa con lo que dice Perines y Murillo (2017), quienes manifiestan que no es una buena práctica investigativa en los centros educativos buscar información para diversos estudios si después no se realiza una retribución real de los hallazgos obtenidos.

Continuando con el diseño pedagógico, se adoptó una práctica que va alineada con el programa de Administración de Empresas, en la cual se realizan procesos de consultoría científico-técnica desde el aula de clase dirigidos al sector empresarial. Por tal razón, no se pensó en un software que simulara una empresa ficticia con un caso empresarial recreado, por el contrario, se pensó en analizar empresas reales de diversos sectores económicos.

Como parte del diseño pedagógico se estructuró la experiencia de aprendizaje empleando la metodología activa del Aprendizaje Basado en Proyectos (ABP) porque fomenta la participación y el trabajo autónomo (Arias y Fidalgo, 2013 citado en Palazuelos, San-Martín, Montoya y Fernández, 2018). El estudiante obtiene la información de la empresa, la incluye al software, este le ayuda en su análisis y obtiene un resultado. El ABP requiere de un producto al final del periodo académico que evidencie la adquisición de la competencia por parte del estudiante, que en este caso se materializa en un documento de consultoría estratégica a la empresa avalado por el profesor.

Toda la estrategia se fundamenta en el aprendizaje situado y su elección se debe a la facilidad de integrar el trabajo de aula con la vida real. Según Tvenge et al, (2016) el desarrollo del conocimiento a través de ambientes situados es un elemento clave para una buena vida laboral y le permite al estudiante mejorar los conocimientos, habilidades y competencias dentro de un ámbito personal, cívico, social y de mutuo intercambio.

\section{Revisión de literatura}

Los AI favorecen el aprendizaje basado en proyectos y permiten mayor efectividad en la formación por competencias, fomentan la creatividad en los estudiantes y mejoran su satisfacción (Arias y Fidalgo, 2013 citado en Palazuelos, San-Martín, Montoya y Fernández, 2018). Además, en una etapa inicial, las instituciones educativas pueden desarrollar AI con base en herramientas de software libre sin necesidad de tener conocimientos 
especializados (Hernández, 2010). Estas facilidades ponen de presente la necesidad de establecer mecanismos de control de calidad en los AI que se divulgan por internet respetando los derechos de autor (Báez et al., 2012). La decisión de emplear un AI, depende de los criterios de usabilidad, intuición y aplicación rigurosa de los conceptos teóricos (Neri et al., 2011).

Alfalla, Medina y Arenas (2011) establecen que el empleo de aplicaciones interactivas multimedia, simuladores o plataformas virtuales en las actividades no presenciales favorece el proceso de aprendizaje a través de la mejora de las variables subjetivas analizadas. Por su parte, León y Cañas (2014) encontraron que, con la aplicación de herramientas de simulación en la Escuela de Administración de Negocios de la Universidad de Costa Rica, los estudiantes no solo se apropiaron la técnica, sino que se sintieron más seguros y más retados con estas herramientas. En contraste, también encontraron barreras de parte de los profesores que les cuesta reemplazar las clases magistrales por métodos activos de enseñanza. González, Padilla y Rincón (2012) consideran que los AI permiten la interacción con los estudiantes de manera dialógica, crítica y reflexiva lo que a su vez fortalece en ellos el desarrollo de competencias humanísticas. Mientras que Cabarcas, Martelo y Tovar (2013) encuentran que el uso de simuladores en los procesos de consultoría facilita la labor operativa en la construcción de matrices y permiten profundizar en el análisis y la toma de decisiones, con lo cual se logra entregar a la empresa productos consultoría de mejor calidad.

En un campo de aplicación similar de la ingeniería industrial, denominado teoría de la manufactura esbelta, Badurdeen, Marksberry, Hall, \& Gregory (2010) encuentran evidencia de las oportunidades de cambio de rol, por parte del tutor, al implementar AI enmarcados en la metodología de ABP. En ese sentido, ese cambio de rol promueve la autonomía del estudiante al contar con una herramienta de acompañamiento permanente a lo largo de todo su proceso de aprendizaje (Martín, Martín \& González Calero, 2007).

Como afirma Saad (2013), la simulación es un método eficaz de aprendizaje de diversos aspectos empresariales ya que llevan al estudiante a comprometerse con sus decisiones a medida que avanza en el proceso, le permite experimentar comportamientos y adquirir habilidades y competencias. Bianchi, Biyona y Landriscina (2000), afirman que la simulación permite articular conocimientos de diferentes áreas de la empresa; motiva al aprendizaje activo; combina el pensamiento estratégico y analítico, fortalece la habilidad de trabajar en equipo y mejorar la comunicación personal y el trabajo bajo presión.

Otro tipo de simulación que existe en pensamiento estratégico se conoce como simulación de vuelo estratégico (SVE); fue pensada para empresas que necesitan despegar de cero. Tales simuladores son de utilidad para el emprendedor en el momento de evaluar y sensibilizarse en el diseño y la determinación de la estrategia más 
conveniente para su nueva empresa (Vidal y Domengue, 2017).

\section{Método}

Para este proyecto se trabajó la implementación de dos AI que permiten mayores interacciones entre usuario y sistema. La estrategia se centró en una alta implicación del estudiante y lo más novedoso del proyecto es que las herramientas son un desarrollo propio de la IUPG. Lo anterior significa que la idea, diseño y estructuración es conducida conceptualmente por los docentes y el LEAI asesora pedagógicamente, acompaña la redacción de los guiones, realiza el diseño gráfico, la animación 3D y la programación. Cuando se termina la herramienta, se realiza la verificación y las pruebas antes de ser llevadas a los estudiantes. Para el desarrollo se emplea la metodología ADDIER que corresponde a las siglas: Análisis (Analysis), Diseño (Desing), Desarrollo (Development), Implementación (Implementation), Evaluación (Evaluation) e Investigación (Research). Esta metodología es propia del laboratorio y sirve para el desarrollo de diversos AI. Es derivada del Modelo de Proceso Interdisciplinario para Desarrollo de Software Educativo de Simulación (MoPIS) y el Modelo de simulación basados en agentes (MABS) propuesto por Brito (2006).

A continuación, se ilustran las metodologías de análisis estratégico que se aplican en los dos AI:

Metodología empleada en el software de diagnóstico empresarial: para el desarrollo se tuvieron en cuenta diferentes herramientas de diagnóstico empresarial integradas bajo la metodología propuesta por David (2003). El proceso inicia identificando los factores el macroentorno establecidos por Fahey \& Narayanan (1986) mediante el análisis PESTEL (ver interfaz del software en la figura 1), luego continua con el análisis del microentorno con el enfoque de las cinco fuerzas de Porter (Dess y Davis, 1984). En una segunda etapa, se hace un análisis interno con la identificación de las fortalezas y debilidades de la compañía en los aspectos financiero, administrativo, tecnológico, de producción, de mercadeo y de talento humano. A partir de estos análisis se construyen las dos primeras matrices de diagnóstico que son la EFE y la EFI. La matriz EFE permite identificar la posición de la compañía para hacer frente los factores del entorno; si el resultado está por encima de 2,5 significa que la compañía se encuentra en una posición competitiva favorable frente al entorno, de lo contrario, las amenazas que enfrente son mayores a sus oportunidades. Por su parte, la matriz EFI permite identificar la posición interna de la compañía para el éxito de la estrategia; un resultado por encima de 2,5 indica que tiene una posición interna fuerte y que son mayores sus fortalezas que sus debilidades al momento de implementar la estrategia (David, 2003).

Las matrices EFI y EFE son el insumo principal para la construcción de la matriz DOFA; Gache y Gache (2006) afirman que el análisis DOFA es fundamental en la identificación de las estrategias más idóneas según el cuadrante estratégico en el que se ubique. El proceso 
continúa con la aplicación de la matriz SPACE desarrollada por Rowe, Mason y Dickel (1982) en la cual se hace un análisis de la compañía a partir de cuatro criterios: fuerza financiera, ventaja competitiva, estabilidad del ambiente y fuerza de la industria; que luego se grafican en forma de cuadrantes.

El proceso termina con la selección de la mejor estrategia para la compañía usando la Matriz Cuantitativa de Planeación Estratégica (CPE) donde se hace una valoración de las estrategias pertenecientes al cuadrante seleccionado de acuerdo con los resultados de la matriz SPACE. Cada estrategia se evalúa a la luz de las fortalezas y debilidades previamente identificadas, de tal manera que la estrategia con mayor puntuación debe ser implementada por la compañía. Con esto se termina el diagnóstico empresarial.

Figura 1. Software de diagnóstico empresarial

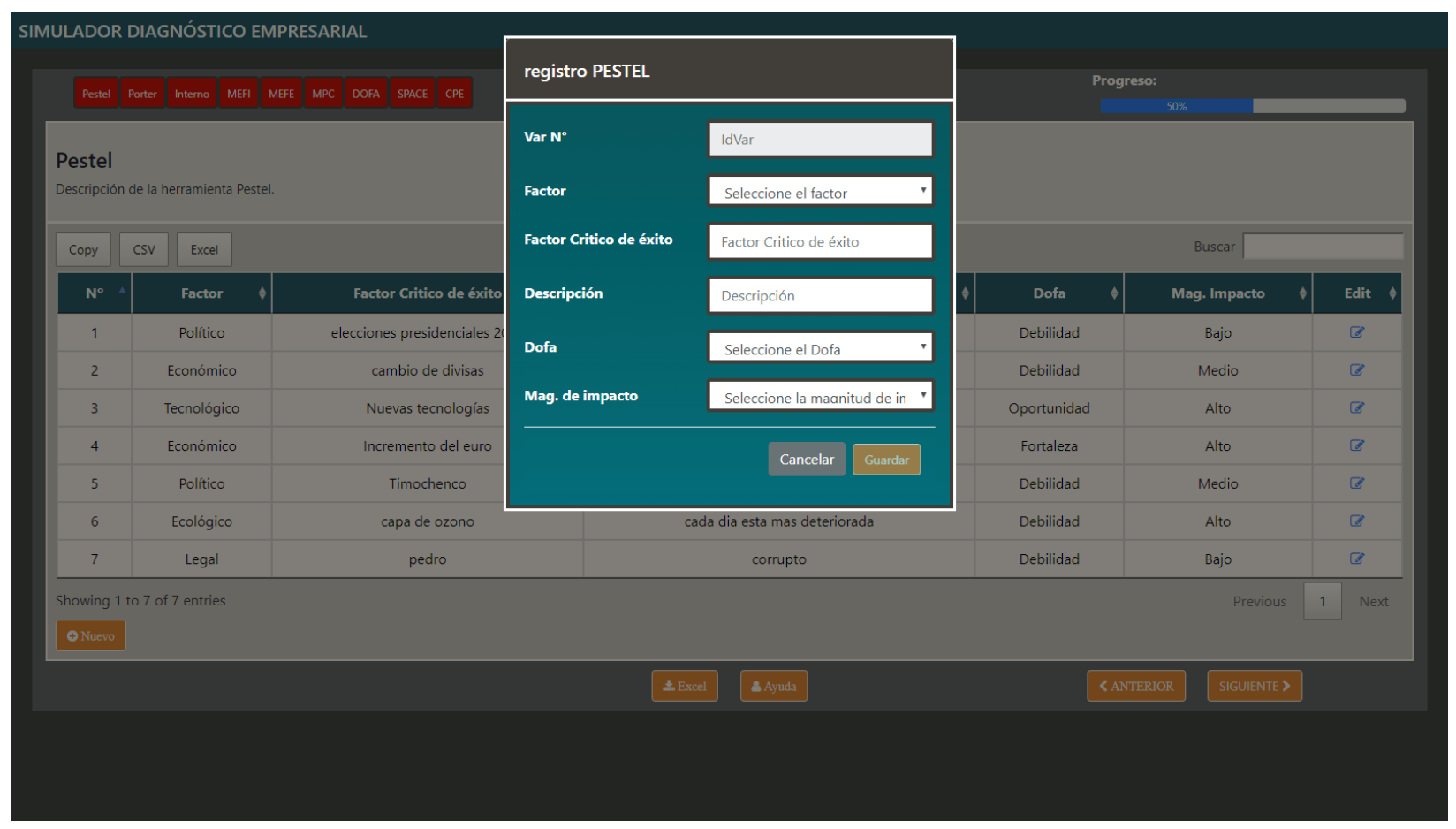

Fuente: elaboración propia (2019).

Metodología empleada en el software de análisis prospectivo: el origen del análisis prospectivo se remonta al año 1945 cuando el Departamento de Defensa de los Estados Unidos crea el proyecto Rand Co. y se crea el laboratorio de ideas que desarrolla las primeras técnicas de análisis del futuro: el método Delphi propuesto por Dalkey \& Helmer (1962) y el análisis morfológico. Sin embargo, es en Francia donde se desarrolla y estructura el concepto de análisis prospectivo con el aporte de De Jouvenel (1972), en donde manifiesta que existen muchos futuros posibles denominados futuribles. Una década después, esta escuela se fortalece con las ideas de Godet (2000) donde se combinan los conceptos de prospectiva con la planeación estratégica; tal vez su mayor 
aporte radique en la unificación de herramientas técnicas que dan un sustento metodológico al análisis prospectivo.

Al igual que el diagnóstico empresarial, el análisis prospectivo parte de la identificación de las fortalezas, debilidades, oportunidades y amenazas que enfrenta la compañía. La diferencia radica en que mientras el diagnóstico toma una fotografía del hoy, la prospectiva hace una proyección de factores internos y externos llamados tendencias que potencialmente tendrán un impacto en un horizonte entre 10 y 15 años. Esas tendencias son ingresadas por el estudiante (Ver figura 2 interfaz para el ingreso de variables).

La siguiente etapa consiste en identificar y clasificar las tendencias clave para el negocio, por medio de las matrices de análisis estructural principalmente la de impactos cruzados y el análisis MIC-MAC. La matriz de impactos cruzados permite cuantificar las tendencias de acuerdo con su nivel de dependencia e influencia respecto a las demás variables. La dependencia se refiere a que tanto una variable analizada es afectada por otras. Las influencias evalúan el impacto de la variable a analizar en el resultado de las otras. De la sumatoria de los valores en la matriz se obtienen los puntos del gráfico para el análisis MIC MAC, este gráfico se presenta a través de los siguientes cuadrantes: poder, conflicto, resultado y autonomía; sólo se tienen en cuenta las tendencias estratégicas clave ubicadas en poder y conflicto.

Luego, el estudiante desarrolla las hipótesis o posibles resultados futuros que podrían tener cada una de las tendencias ubicadas en poder y conflicto. El software realiza una serie de combinaciones con las probabilidades de las hipótesis y obtiene los diez escenarios más probables; de allí debe seleccionar el escenario "meta" con el cual desarrollará su planeación estratégica. Tal planeación es la que entrega como proyecto de aula.

Figura 2. Software de análisis prospectivo versión final

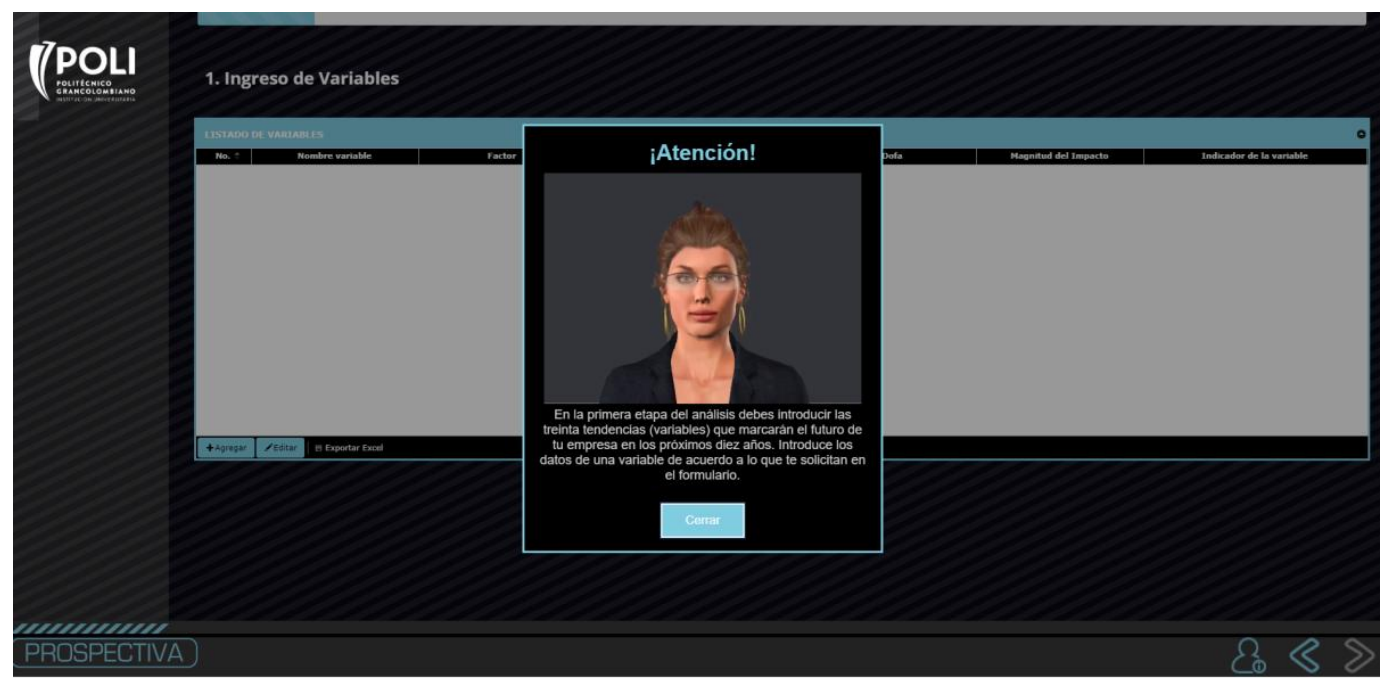

Fuente: Creación propia (2019). 


\section{Elaboración y aplicación de los AI}

La primera fase comprende el uso de aplicativos en versión beta (es decir en versión Excel), la segunda el desarrollo y programación (en .NET y en angular). El piloto fue aplicado en dos cursos presenciales del pregrado de Administración de Empresas de la IUPG. El primer AI se aplicó a una muestra de nueve cursos de diagnóstico empresarial cada uno con 25 estudiantes en promedio. El segundo AI se aplicó a una población similar en el curso de estrategias gerenciales. Se trabajó con estudiantes de la modalidad presencial por la facilidad de retroalimentación y corrección de errores en los aplicativos.

Al finalizar el periodo académico, se aplicó un instrumento diseñado para recoger las percepciones de los estudiantes con respecto al uso de los AI. Respondieron el instrumento 42(19\%) estudiantes de la asignatura Diagnóstico Empresarial y 79 (35\%) de Estrategias Gerenciales. Este instrumento estaba dividido en tres partes, en la primera el estudiante hacia una evaluación general de los AI, en la segunda evaluaron sus atributos y en la tercera las oportunidades de mejora.

\section{Resultados}

Los resultados muestran un alto nivel de satisfacción con el uso de las AI. Un 69,7\% de los estudiantes calificaron por encima de cuatro sobre cinco (4/5) su experiencia con el aplicativo de prospectiva, mientras que lo mismo hizo el $64,3 \%$ con el aplicativo de diagnóstico empresarial.

Tabla 1. Calificación de los estudiantes sobre los aplicativos

\begin{tabular}{ccc}
\hline Calificación & Aplicativo de Prospectiva & Aplicativo de Diagnóstico Empresarial \\
Cinco & $8,90 \%$ & $11,90 \%$ \\
Cuatro & $60,80 \%$ & $52,40 \%$ \\
Tres & $22,80 \%$ & $23,80 \%$ \\
Dos & $7,50 \%$ & $7,10 \%$ \\
Uno & $0,00 \%$ & $4,80 \%$ \\
\hline
\end{tabular}

Fuente: Elaboración propia (2019).

Asimismo, en los dos casos los estudiantes consideraron que los AI fueron una ayuda efectiva para cumplir los objetivos planteados en el análisis empresarial. De igual manera, los estudiantes consideran que los mayores beneficios están en mejor análisis empresarial y que los AI les sirven para un mayor entendimiento del proceso de análisis estratégico. Ver tabla 2. 
Tabla 2. Ventajas percibidas por los estudiantes en los aplicativos

\begin{tabular}{ccc}
\hline Percepción & $\begin{array}{c}\text { Aplicativo } \\
\text { de } \\
\text { Ahorro de Tiempo }\end{array}$ & $\begin{array}{c}\text { Aplicativo de } \\
\text { Diagnóstico } \\
\text { Empresarial } \\
25,00 \%\end{array}$ \\
Minimización de errores procedimentales & $20,78 \%$ & $14,06 \%$ \\
Mejora el análisis que se puede hacer de la compañía & $22,73 \%$ & $25,00 \%$ \\
Ayuda a entender mejor el proceso de análisis estratégico & $27,27 \%$ & $18,75 \%$ \\
Facilita correcciones y ajustes al diagnóstico luego de recibir & $12,99 \%$ & $17,19 \%$ \\
\hline la retroalimentación del tutor & & \\
\hline
\end{tabular}

Fuente: Elaboración propia (2019).

Finalmente, a los estudiantes se les consultó con respecto a los aspectos de mejora que hubieran permitido una experiencia de simulación más amigable para lo cual se encontraron los siguientes resultados:

Tabla 3. Aspectos de mejora en los aplicativos

\section{Percepción}

Mas explicación por parte del tutor con respecto a la forma de usar el aplicativo

Mayor interactividad. Explicaciones y aclaraciones entregadas por el aplicativo en cada etapa de la simulación

La existencia de un manual de usuario para entender mejor su

\section{Aplicativo \\ de \\ Prospectiva \\ Aplicativo de Diagnóstico Empresarial}

$24,43 \%$

$37,40 \%$

$32,82 \%$
$30,77 \%$

$38,46 \%$ manejo

$$
\text { Fuente: Elaboración propia (2019). }
$$

El aspecto más importante para mejorar fue la interactividad y las explicaciones entregadas por el aplicativo en cada etapa de la simulación; seguida, por la existencia de un manual de usuario y de la necesidad de mayor explicación del uso del aplicativo por parte del tutor. Esto se subsanó con animaciones 3D y un avatar que explica paso a paso lo que debe hacer el estudiante, de igual manera, se creó el manual de usuario interactivo y en pdf.

\section{Discusión}

En línea con los hallazgos realizados por Palazuelos, San-Martín, Montoya y Fernández (2018) la mayor parte de los estudiantes que participaron de la prueba encontraron que los softwares fueron una ayuda efectiva para el cumplimiento de los objetivos de aprendizaje propuestos. Tanto para los estudiantes que emplearon el AI de prospectiva y el de diagnóstico empresarial, el aspecto más importante fue que los ayudó a entender mejor el proceso de análisis estratégico. Adicionalmente, también es significativa la percepción favorable en torno a la mejora en la capacidad de análisis, haciendo uso del 
AI.

La diferencia de las herramientas propuestas en este estudio con las de simulación que se encuentran en el mercado, genera una apropiación especial en lo que a pensamiento estratégico se refiere. Las herramientas que se encuentran en el mercado comúnmente le presentan al estudiante una empresa simulada y de acuerdo con las decisiones que se tomen, varían algunos indicadores clave. Es significativo el hecho de que se considere relevante el ahorro de tiempo dado que en principio, uno podría imaginar que los tutores y los estudiantes preferirían evitar el uso de la herramienta, por la inversión de tiempo en el aprendizaje y apropiación.

De otro lado, el pensamiento estratégico se trabaja desde cómo planear una jugada que le permita obtener buenos resultados en el simulador. En cambio, en este proceso se realiza un análisis de una empresa real, con condiciones reales, el software de diagnóstico arroja un resultado de la situación actual de la empresarial, y en prospectiva, se define el escenario meta. A partir de esa información, el estudiante debe construir el plan estratégico a seguir. En este caso el pensamiento estratégico se basa en la construcción de matrices y análisis acordes con la realidad que afronta la empresa, que son el soporte de su proceso de consultoría.

La planeación estratégica consta de 4 fases, el análisis externo, el análisis interno, el diseño de la estrategia y la implementación. Como ya se trató en este artículo, el software de diagnóstico ayuda en las dos primeras etapas y el estudiante desarrolla una tercera con el plan estratégico a seguir. La cuarta dedicada a la implementación del plan no se puede evidenciar por la duración del curso.

\section{Conclusiones}

El AI les permitió a los estudiantes confrontar los conocimientos adquiridos en el programa con un escenario real. Esto significa un proceso de pensamiento estratégico más profundo porque el estudiante debe afrontar las particularidades de cada caso.

Los AI diseñados en este proyecto son herramientas para aplicación directa en cualquier empresa de cualquier sector de la economía, sin importar si es nueva o ya tiene una experiencia, y se hace análisis estratégico desde una óptica perspectiva (diagnóstico) y una óptica prospectiva (análisis prospectivo). A diferencia del SVE que sólo se aplica cuando se pretende iniciar una empresa.

Un factor de éxito de la estrategia es dado por el acompañamiento y asesoramiento del tutor. Una capacitación adecuada y la participación activa en la implementación de lo AI constituye un elemento esencial para la construcción de una experiencia de aprendizaje en el estudiante; en concordancia con lo que afirma León y Cañas (2014) relacionado con la dificultad de los profesores para adoptar metodologías de enseñanza más lúdicas, uno de los aspectos de mejora de mayor importancia identificado por los estudiantes, fue la 
necesidad de una mayor explicación de los profesores con respecto al uso del aplicativo.

La mayor parte de los estudiantes percibieron que obtuvieron un ahorro de tiempo importante en el desarrollo de los análisis estratégicos, lo cual coincide con lo observado por Cabarcas, Martelo y Tovar (2013) en relación con las facilidades operativas que permiten el uso de los simuladores en los procesos de consultoría; este ahorro de tiempo es importante en la medida que puede ser utilizado en fortalecer los procesos de análisis y la calidad de los documentos de consultoría entregados.

Con los AI aplicados en este proyecto se evidencia el mejoramiento de los resultados de los estudiantes y cómo un software centrado en la didáctica facilita el proceso de aprendizaje en diversos aspectos como: eficiencia en la autorregulación y gestión del estudiante (ahorro de tiempo, minimización de errores procedimentales), mayor profundidad en la apropiación del conocimiento (mejora el análisis que se puede hacer de la compañía), mayor adquisición de la competencia (comprender mejor el proceso de análisis estratégico); mejor adaptación al proceso personal de aprendizaje (facilita correcciones y ajustes al diagnóstico); mayor relación dialógica entre estudiante y tutor (recibir la retroalimentación). Estas ventajas y otras encontradas en otros estudios señalados en este documento permiten evidenciar que el uso de estrategias de aprendizaje inmersivo en estudiantes de administración es un potenciador para su desempeño académico y profesional, lo cual indica que es factible implementar estrategias similares para otros cursos del programa.

\section{Referencias}

Alfalla, R., Medina, C. \& Arenas, F. (2011). Mejorando la formación en Dirección de Operaciones: la visión del estudiante y su respuesta ante diferentes metodologías docentes. Cuadernos de Economía y Dirección de la Empresa, 14(1), 40-52. Doi: https://doi.org/10.1016/j.cede.2011.01.002

Badurdeen, F., Marksberry, P., Hall, A., \& Gregory, B. (2010). Teaching lean manufacturing with simulations and games: A survey and future directions. Simulation \& Gaming, 41(4), 465-486.Doi: https://doi.org/10.1177/1046878109334331

Báez, C. P., Manzouli, C. H., Cifuentes, Y. S., Gómez, D. D., Duarte, M. S., Otero, M. P., y Rees, G. P. (2012). Alfabetización informacional en la educación superior virtual: logros y desafíos. Información, cultura y sociedad, (26), 83-104.

Bianchi, C., Bivona, E. y Landriscina, F. (2000). Promoting Entreprenership Through Open- 
Distance-Learning Management Flight Simulators EcoRoll Educational Package. International System Dynamics Conference, Society, Bergen.

Brito, J. (2006). Gestión del Proceso de Desarrollo de Simuladores Virtuales Educativos un $\begin{array}{llll}\text { enfoque } & \text { transdisciplinario. } & \text { Recuperado }\end{array}$ http://sedici.unlp.edu.ar/bitstream/handle/10915/19191/Documento_completo.pdf?seq uence $=1$

Cabarcas, A., Martelo, R. y Tovar, Luis. (2013). Software para mejorar la aplicación de técnicas cuantitativas en estudios prospectivos. Cuadernos de Administración, 29(49), 64-74. Recuperado de https://dialnet.unirioja.es/descarga/articulo/5006550.pdf

Dalkey, N. \& Helmer, O. (1962). An Experimental Application of the Delphi Method to the Use of Experts. Recuperado de https://www.rand.org/content/dam/rand/pubs/research_memoranda/2009/RM727.1.pd $f$

David, R. (2003). Conceptos de Administración Estratégica. México: Pearson.

Dede, C. (2009). Immersive interfaces for engagement and learning. science, 323(5910), 66-69. Doi: https://doi.org/10.1126/science.1167311

De Jouvenel, H. (1972). Invitation a la prospective. Paris: Edition futuribles.

Dess, G \& Peter. D.(1984). Porter's (1980) Generic Strategies as Determinants of Strategic Group Membership and Organizational Performance. The Academy of Management Journal, 27(3), 467-488. Recuperado de https://www.jstor.org/stable/256040

Fahey, L. \& Narayanan, K. (1986). Macroenvironmental Analysis for Strategic Management. St. Paul, Minnesota: West Publishing Company.

Franco, M., Silva, R. \& Rodrigues, M. (2019). Partnerships between higher education institutions and firms: The role of students' curricular internships. Industry and Higher Education, 33(3), 172-185. Doi: https://doi.org/10.1177/0950422218819638

Gache, F. \& Gache, D. (2006) Propuesta de modelos para captar las influencias del 
entorno en la evaluación de alternativas para las PyME. Recuperado de https://www.academia.edu/2112291/PROPUESTA_DE_MODELOS_PARA_CAPTAR_LAS_ INFLUENCIAS_DEL_ENTORNO_EN_LA_EVALUACI\%C3\%93N_DE_ALTERNATIVAS_PARA _LAS_PYME?auto=download

Godet, M. (2000). La Caja de Herramientas de la Prospectiva Estratégica. Recuperado de http://es.laprospective.fr/dyn/espagnol/bo-lips-esp.pdf

González, K., Padilla, J. \& Rincón, D. (2012) Formación del docente en contextos blearning: implicaciones tecnológicas, investigativas y humanísticas. Revista Virtual Universidad Católica del Norte (36), 48-74. Recuperado de https://revistavirtual.ucn.edu.co/index.php/RevistaUCN/article/view/371

Hernández, R. (2010). Educative use of simulators in free software for the education of the physics in the engineering programs. IEEE Education Engineering Conference, EDUCON(2010), 621-628. Doi: https://doi.org/10.1109/EDUCON.2010.5492521

Klink, M. Van der. \& Boon, J. (2002). The investigation of competencies within professional domains. Human Resource Development International, 5(4), 411-424.

León, E. \& Cañas, F. (2014). Modelos de simulación en la Escuela de Administración de Negocios. Revista de las Sedes Regionales, 15(31), 86-98. Recuperado de https://revistas.ucr.ac.cr/index.php/intersedes/article/view/16015/15416

Martín, G., Martín, G., \& González Calero, A. (2007). Active learning in interactive simulations. Inteligencia Artificial, 11(33), 25-36. Recuperado de https://www.scopus.com/record/display.uri?eid=2-s2.036049047805\&origin=inward\&txGid=a26aa5096b28f91744108e6a8aba63d6

Neri, L., Noguez, J., Pérez, I. \& Aguilar, G. (2011). Facilitating the design of physics active learning problems through authoring simulation tools: Authorphysics. Frontiers in Education Conference (FIE), (2011), S3C-1-S3C-6. Doi:

https://doi.org/10.1109/FIE.2011.6143085

Palazuelos, E., San-Martín, P., Montoya del Corte, J. \& Fernández-Laviada, A. (2018). Utilidad percibida del Aprendizaje Orientado a Proyectos para la formación de 
competencias. Aplicación en la asignatura «Auditoría de cuentas». Revista de Contabilidad-Spanish Accounting Review, (21), 150-161. Doi: https://doi.org/10.1016/j.rcsar.2017.04.004

Perines, H. \& Murillo, J. (2017). Percepciones de los docentes en formación sobre la investigación educativa. Estudios pedagógicos (Valdivia), 43(1), 251-268. https://dx.doi.org/10.4067/S0718-07052017000100015

Rowe, A. J., Mason, R. O., Dickel, K., Mann, R. \& Mockler, R. (1982). Strategic Management: A Methodological Approach. United States: Addison-Wesley.

Saad, F. (2013). Modeling and comparing a startup dynamic in the US and Egypt. Recuperado de https://dspace.mit.edu/handle/1721.1/90701

Smyth, J. \& Dow, A. (1998). What's wrong with outcomes? Spotter planes, action plans, and steerage of the educational workplace. British Journal of Sociology of Education, 19(3), 291-303.

Stapleton, R. J. (1990). Academic entrepreneurship: Using the case method to simulate competitive business markets. Organizational Behavior Teaching Review, 14(4), 88104.

Tchibozo, G. (2008). Extra-curricular activity and the transition from higher education to work: A survey of graduates in the United Kingdom. Higher Education Quarterly, 61(1), 37-56. Doi: https://doi.org/10.1111/j.1468-2273.2006.00337.x

Téllez, L. (2005). Pensamiento estratégico y desarrollo de competencias gerenciales: una perspectiva desde las unidades de información. Revista Códice, (2), 115-123. Recuperado de https://core.ac.uk/download/pdf/17184113.pdf

Tobón, S. (2006). Competencias en la educación superior: Políticas hacia la calidad. Bogotá: Ecoe Ediciones.

Tvenge, N., Martinsen, K., Vijay, S. \& Kolla, K. (2016). Combining Learning Factories and ICT- based Situated Learning. Procedia CIRP, (54), 101-106. Doi: https://doi.org/10.1016/j. procir.2016.03.031 
Vidal, D. \& Muñoz, R. (2017). Simulador de vuelo ejecutivo como medio de aprendizaje en la planeación de recursos de nuevas empresas bajo el enfoque del marcador balanceado. Revista Contaduría y Administración Volumen, 62(2), 556-576. https://doi.org/10.1016/j.cya.2017.01.006

\section{Agradecimientos}

A la IUPG por la implementación de un Laboratorio de Experiencias de Aprendizaje Inmersivo que permite la elaboración de proyectos basados en TIC con experiencias de simulación y aplicativos a partir de las necesidades que tienen los docentes en las aulas. A la Facultad de Ciencias Administrativas Económicas y Contables y su decana Deisy de la Rosa por el respaldo y confianza depositada en este proyecto. A la gerente de Desarrollo Virtual María del Socorro Guzmán. 